\title{
Advances in cancer pain from bone metastasis
}

This article was published in the following Dove Press journal:

Drug Design, Development and Therapy

18 August 2015

Number of times this article has been viewed

\author{
Xiao-Cui Zhu' \\ Jia-Li Zhang' \\ Chen-Tao Ge' \\ Yuan-Yang Yu' \\ Pan Wang' \\ Ti-Fei Yuan ${ }^{2}$ \\ Cai-Yun $\mathrm{Fu}^{1,3}$ \\ 'College of Life Sciences, Zhejiang \\ Sci-Tech University, Hangzhou, ${ }^{2}$ School \\ of Psychology, Nanjing Normal \\ University, Nanjing, ${ }^{3}$ Institute for Cell- \\ Based Drug Development of Zhejiang \\ Province, Hangzhou, People's Republic \\ of China
}

\begin{abstract}
With the technological advances in cancer diagnosis and treatment, the survival rates for patients with cancer are prolonged. The issue of figuring out how to improve the life quality of patients with cancer has become increasingly prominent. Pain, especially bone pain, is the most common symptom in malignancy patients, which seriously affects the life quality of patients with cancer. The research of cancer pain has a breakthrough due to the development of the animal models of cancer pain in recent years, such as the animal models of mouse femur, humerus, calcaneus, and rat tibia. The establishment of several kinds of animal models related to cancer pain provides a new platform in vivo to investigate the molecular mechanisms of cancer pain. In this review, we focus on the advances of cancer pain from bone metastasis, the mechanisms involved in cancer pain, and the drug treatment of cancer pain in the animal models.
\end{abstract}

Keywords: cancer pain from bone metastasis, animal models of cancer pain, molecular mechanisms, drug treatment

\section{Introduction}

The World Health Organization published the "World Cancer Report 2014" on September 3, 2014, reporting that cancer is a leading cause of death worldwide, and the total number of cancer incidence and mortality globally is increasing disturbingly in 2012, and that nearly half of the new cancer cases occurred in Asia, in which Chinese population's cancer rate is the first. The report predicts that global cancer cases will increase rapidly from 14 million in 2012 to 19 million in 2025 and to 24 million in $2035 .^{1}$

With the improvement of cancer treatment, the 5-year survival rate of patients with cancer has been greatly improved. The cancer pain that comes along with cancer seriously affects the quality of life of patients. Statistically, approximately $60 \%-90 \%$ of patients with advanced cancer have been tortured by varying degrees of pain, of which approximately $30 \%$ of patients have been tortured by persistent severe pain. ${ }^{2}$ Bone cancer pain is the most common form of pain for a patient with cancer, and the major reason for this kind of pain is due to the cancer metastasis to bone and then the invasion of surrounding soft tissue violations. ${ }^{3}$ Two-thirds of patients with advanced cancer are prone to bone metastases. The bone metastases are also considered as the most common reason for cancer pain..$^{4-7}$ During the bone metastasis, the metastases to the bone are most vulnerable in lung, breast, prostate, and ovarian cancers. ${ }^{8}$

Although there are a variety of methods applying to treat cancer pain, such as bisphosphonates, chemotherapy, surgery, nerve block, adoptive tumor immunotherapy, and gene knockout, the clinic treatment of cancer pain is still to focus on the three-step program, which is established by the World Health Organization. According to the degree of pain, the patients will be given a nonsteroidal anti-inflammatory analgesics (mild pain) and/or opioid therapy (moderate and severe pain). However, many patients tortured by cancer pain could still not been controlled appropriately, and there are many 
problems needed to be solved now, such as "mirror pain", morphine tolerance, constipation, respiratory depression for opioid drugs, and stomach ulcers and kidney toxicity for nonsteroidal anti-inflammatory analgesics. ${ }^{9}$ The clinical use of these drugs could be limited by these side effects. ${ }^{10}$ Due to the fact that the molecular mechanisms of bone cancer pain have not been elucidated, and that the side effects and tolerability of clinical available drugs cannot be overcome, so $45 \%$ of patients with cancer accompanied by pain cannot be effectively controlled. ${ }^{11}$ It is imminent and important to elucidate the molecular mechanisms of bone cancer pain and to find efficient ways to improve the life quality of patients with cancer. This review focuses on the animal model of bone cancer pain, research progress in the pathogenesis, and drug treatment of cancer pain.

\section{The establishment of animal models of bone cancer pain}

Schwei et $\mathrm{al}^{5}$ first reported the mouse femur pain model in 1999, in which $10^{5}$ NCTC2472 fibrosarcoma cells were implanted into the distal femoral medullary cavity of $\mathrm{C} 3 \mathrm{H} / \mathrm{HeJ}$ mice via incision operation of the mouse left knee. After the postimplantation day of 14 , it arised spontaneous pain and allodynia in behavior, and after the postimplantation day of 21 , there was a significant bone destruction; bone tissue sections were studied using X-ray diffraction. ${ }^{5}$ It is likely that the degree of pain behavior may be related to bone destruction. The pain behavior of these bone cancer mice is similar to that of patients with clinical bone cancer. ${ }^{12}$ Construction of the first mouse model of femoral cancer pain has laid a good animal foundation for the investigation of the mechanisms of cancer pain in animal models. The bone tissue damage, pain behavior, and neurochemical changes in the spinal cord caused by tumor metastasis are usually investigated using this mouse femur pain model. ${ }^{13}$

The mouse calcaneus cancer pain model was established in 2001 via implantation of NCTC2472 fibrosarcoma cells into mouse heel. ${ }^{14}$ Compared with the femur cancer pain mouse model, the abnormal changes of swelling calcaneus in the calcaneus cancer pain mouse model can be visually observed by the naked eye directly. The pain behavioral indicators appear relatively early. The tumor cells implanted into the heel bone in the first 3 days began to grow adhesions, and then osteolysis first appeared in 6 days, accompanied by mechanical hyperalgesia and cold stimuli hyperalgesia, as well as significant bone tissue damage occurred on the 12th day. ${ }^{14}$ Morphine $\left(\mathrm{ED}_{50} 9.0 \mathrm{mg} / \mathrm{kg}\right)$ can significantly relieve the hyperalgesic phenomena in this calcaneus cancer pain mouse model. ${ }^{14}$ The establishment of the calcaneus cancer pain mouse model provides a unique quantified model to investigate the relationship between tumor and pain neural interactions. The research team injected the fibrosarcoma cells into mouse humerus in 2003 to establish the mouse humerus cancer pain model again. All of these mouse cancer pain models will be useful to clarify the mechanisms of bone metastases of cancer pain in the central and peripheral regions and also useful to evaluate the efficacy of analgesics in different types of cancer pain. ${ }^{15}$

Medhurst has established the tibia bone cancer pain model in rats in 2002 by injecting $3 \times 10^{3}$ or $3 \times 10^{4}$ MRMT- 1 breast cancer cells into tibia bone marrow cavity of homologous Sprague Dawley rats. The results of X-ray analysis showed that the tibia bone is significantly damaged after 10-14 days, and that the bone mineral content and density decreased after 20 days, as well as the number of osteoclasts increased significantly, which is examined by the tartrate-resistant acid phosphatase staining of histological sections, accompanied by allodynia and hyperalgesia. ${ }^{16}$ Subcutaneous morphine (1-3 mg/kg) can reverse these pain behaviors. ${ }^{16}$ Radiological and histological examination showed local tumor growth, but no tumor cells spread to other parts. ${ }^{16}$ Morphine dose dependently attenuated mechanical hyperalgesia induced by MRMT-1 cell transplantation, but cyclooxygenase-2 (COX-2) inhibitor celecoxib produced no mitigation effect in this pain model, suggesting that prostaglandin (PG) pain factors may not be involved in this kind of pain model. ${ }^{16,17}$ The operation of rat tibia bone cancer pain model is simple, and this model is of great significance to the study of bone cancer pain.

In humans, bone destruction caused by tumor can induce a dull pain. Accompanied with the growth of tumor cells and the destruction of bone, the pain gradually strengthens, in which mild compression or position change can induce intense pain in consistent with the degree and frequency of bone destruction. ${ }^{18}$ The establishment of several kinds of rodent bone cancer pain model from 1999 could reproduce the symptoms of patients with bone cancer pain to a certain extent. Using the animal model of bone cancer pain, researchers can better understand the molecular mechanism of bone cancer pain and can develop new drugs for the treatment of bone cancer pain.

\section{The mechanisms of bone cancer pain}

Bone cancer pain is a kind of chronic pain with unique and complicated mechanisms. In mouse/rat bone cancer pain 
models, the pain behaviors such as spontaneous pain, hyperalgesia, and allodynia pain are related to the neurochemical changes at the spinal cord level accordingly, including substance P, c-Fos, and Dynorphin expression. The hyperalgesia induced by cancer includes central sensitization mechanisms and peripheral sensitization. ${ }^{19,20}$ Studies have shown that bone cancer pain may be related to algogenic substances produced by tumor cells and inflammatory cells, as well as sustained activation of osteoclasts and nerve compression and injury caused by tumor growth. ${ }^{19}$

\section{Dissolve bone}

Clinical analysis of bone metabolism in patients with bone metastases showed tumor-induced bone destruction (osteolysis) is closely related to the occurrence of cancer pain. Studies in animal models confirmed that osteoclasts can be activated by tumor necrosis factor (TNF) and interleukin secreted by cancer cells to dissolve the bone, and the extent of damage and pain was positively correlated with the neurochemical changes of spinal dorsal root ganglia (DRG). Studies have shown that the signaling pathway of receptor activator of nuclear factor- $\kappa \mathrm{B}$ (RANK) plays a critical role in this process. RANK ligand (RANKL), a membrane-bound protein expressed primarily on the surface of osteoblasts and bone marrow stromal cells, binds to RANK on the surface of osteoclast precursors, stimulating their differentiation into mature osteoclasts. ${ }^{21}$ Bone-resorbing osteoclasts secrete protons to degrade bone minerals and form a highly acidic microenvironment. Then, the acidic microenvironment directly excites sensory neurons innervating bone via activation of the acidsensing nociceptors, transient receptor potential vanilloid 1, and acid-sensing ion channel 3, resulting in bone cancer pain eventually. $^{22}$

\section{Peripheral sensitization}

Primary sensory neurons located in the DRG can be divided into two general types: A-fiber and C-fiber. Thickly myelinated $A-\beta$ fibers can conduct the non-noxious stimulation, whereas thinly myelinated $A-\delta$ fibers and peptidergic $C$ fibers are predominant sensory neurons innervating the bone with various receptors to feel different stimulation, such as transient receptor potential vanilloid 1, cold receptor (coldand menthol-sensitive receptor), transient receptor potential melastatin 8 , mechanically gated ion channel $\mathrm{P} 2 \mathrm{X} 3$ receptor, endothelin (ET) receptor, and PG receptor. A variety of noxious stimulation can be converted to electrochemical signaling by these receptors, and these electrochemical could be transmitted to the central nervous system (CNS). Under continuous peripheral stimulation, the plasticity of DRG neurons could be changed, the sensitivity of peripheral nerve could be increased, and pain threshold could be lowered, resulting in hyperalgesia. In the process of the development of bone cancer, the tumor tissue can secrete a series of neurochemicals, such as TNF- $\alpha$, PG, ET, epidermal growth factor, transforming growth factor, and platelet-derived growth factor- $\beta$. These factors activate and sensitize corresponding receptors to excite primary afferent neurons..$^{20,23-25}$

It has been confirmed that ET-1 is likely to be involved in cancer pain. ${ }^{23,24}$ In the calcaneus mouse model, the ET-1 levels in tumor tissue fluid increased after injecting tumor cell 7-12 days. In addition, the ET-1 levels in tumor tissue homogenate and sarcoma cells in vitro assays were also increased, which was consistent with the in vivo results. ${ }^{23,24}$ Injection of ET-1 to mouse plantar increased spontaneous pain behavior, and injection of $\mathrm{ET}_{\mathrm{A}}$ receptor antagonist could partially block the pain behavior. ${ }^{14}$ Furthermore, there was high concentration of ET-1 secretion in patients with metastatic breast cancer, suggesting that ET-1 may be involved in the process of cancer pain. ${ }^{8}$

In addition, $\mathrm{PG}$ also plays an important role in bone cancer pain. To synthesize a large number of $\mathrm{PG}$, tumor tissue can express high levels of COX-2. ${ }^{26}$ Sabino et $\mathrm{al}^{26}$ reported that selective COX-2 inhibitors (intraperitoneal, i.p.) could significantly relieve cancer pain behaviors in mouse femoral pain model and significantly decrease the expression levels of c-fos and dynorphin, suggesting that PGs may play an important role in cancer pain and that the mechanism may be related to the PG synthesized by the tumor cells combined to the $\mathrm{PG}$ receptors expressed in nociceptors participated in the nociceptors sensitization and/or activation.

\section{The central sensitization}

The CNS of bone cancer pain animals had significant pathological changes related to the generation and maintenance of

Table I The drugs and effects for bone cancer pain ${ }^{20,22,29}$

\begin{tabular}{|c|c|}
\hline Drug & Effects \\
\hline $\begin{array}{l}\text { Nonsteroidal anti-inflammatory } \\
\text { drugs }\end{array}$ & $\begin{array}{l}\text { Inhibiting the activities of } \\
\text { cyclooxygenase (COX) }\end{array}$ \\
\hline Opioids & Activation of opioid receptors \\
\hline Bisphosphonates & $\begin{array}{l}\text { Inducing cancer cell proliferation, } \\
\text { inhibiting osteoclast activity }\end{array}$ \\
\hline Osteoprotegerin (OPG) & $\begin{array}{l}\text { Inhibiting the binding of RANKL to } \\
\text { RANK on the surface of osteoclast }\end{array}$ \\
\hline $\begin{array}{l}\text { Endothelin receptor } \\
\text { antagonists }\end{array}$ & $\begin{array}{l}\text { Blocking tumor activation to } \\
\text { peripheral receptors }\end{array}$ \\
\hline
\end{tabular}

Abbreviations: RANKL, receptor activator of nuclear factor- $\mathrm{KB}$ ligand; RANK, receptor activator of nuclear factor- $\mathrm{KB}$. 


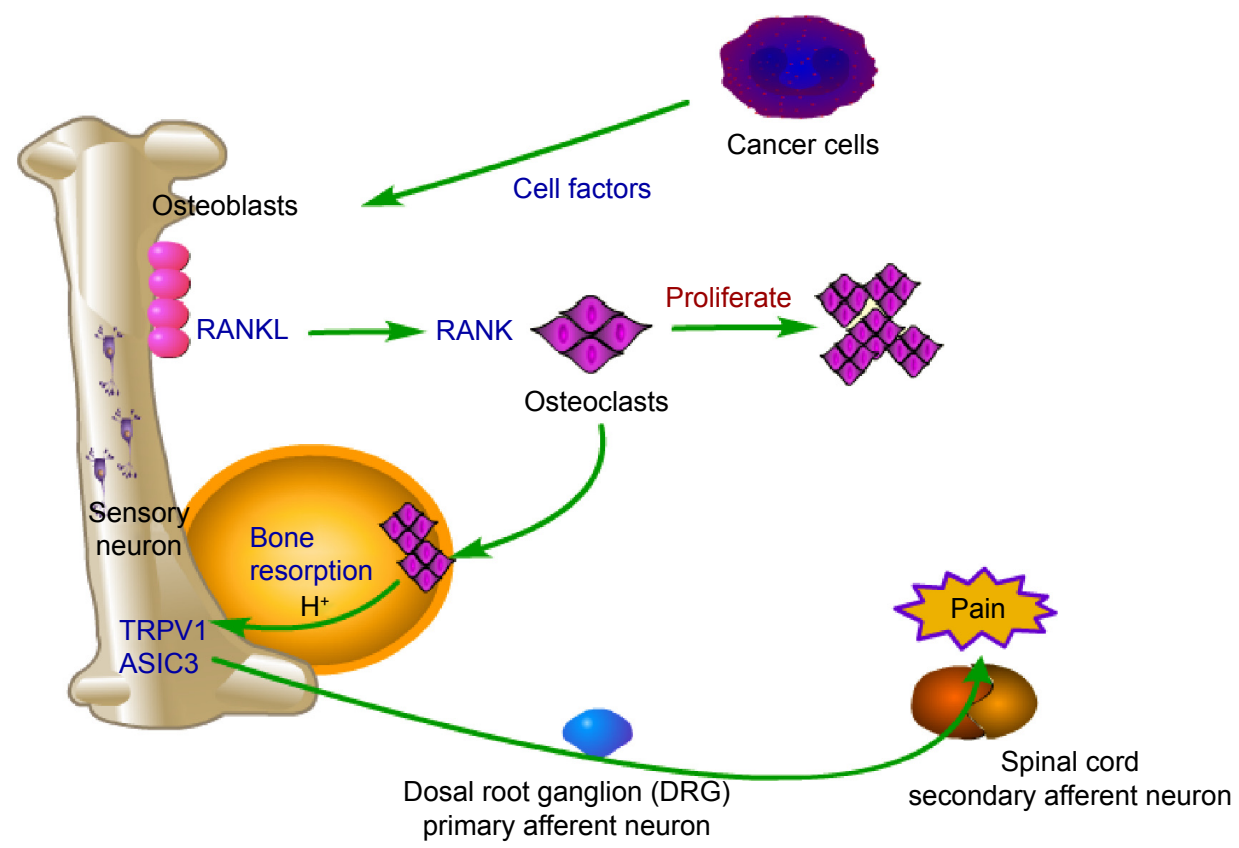

Figure I Mechanism of bone cancer pain.

Notes: In the process of bone cancer pain, cancer cells secrete diverse cell factors to promote proliferation and stimulate osteoclastic bone resorption via activation of receptor activator of the nuclear factor-kB (RANKL)/RANK pathway in osteoblasts and osteoclasts. Bone-resorbing osteoclasts secrete protons to degrade bone minerals and form a highly acidic microenvironment. Then, the acidic microenvironment directly excites sensory neurons innervating bone via activation of the acid-sensing nociceptors, TRPVI (transient receptor potential vanilloid I) and ASIC3 (acid-sensing ion channel 3), transducing noxious signals via DRG (primary afferent neuron), and spinal cord (secondary afferent neuron) and evokes bone pain in brain.22

Abbreviation: DRG, dorsal root ganglia.

cancer pain. ${ }^{27}$ Previous studies reported the neurochemical changes in the corresponding segments of the spinal cord in the bone cancer pain mice including hyperplasia and hypertrophy of astrocytes, transporter reduction of glutamate reuptake, and the expression increase of dynorphin and c-Fos expression. Non-noxious stimulation could induce the decrease of pain threshold in mouse bone cancer pain models, as well as the neurochemical changes, while only the nociceptive stimulation could induce these changes in normal mice, suggesting the presence of peripheral afferent nerve sensitization in mouse bone cancer pain models. ${ }^{5,16,20,28}$ The astrocytes can release various cytokines and growth factors to change the chemical composition of peripheral nerves, in which the elevated extracellular glutamate due to the decrease of glutamate transporter could produce excitotoxicity of the CNS, suggesting that the change of neurochemicals in spinal cord may be involved in cancer pain sensitization in the CNS. $5,16,20,28$

\section{The direct effect of tumor cells to peripheral nervous system}

Sensory nerve fibers and sympathetic nerve fibers are less distribution in the tumor itself but more distribution in the periosteum. In the latter developmental process of the cancer, the pain occurred due to the traction of periosteum and the oppression of the nerve fiber in periosteum induced by the rapid growth of the tumor. In addition, cytokines, growth factors, and other chemicals secreted by the cancer cells can damage the structure of local tissue or the distribution of nerve fiber, causing the incidence of bone cancer pain. ${ }^{29}$

\section{Pathological fracture}

Bone fractures may be occurred in the patients with bone cancer metastases. In daily activities, tumble or injuries may induce fractures, and the thinned bones are also very prone to be broken. ${ }^{30}$ The bone is the most important structure to maintain the movement. The pain in patients with cancer with bone metastases also includes the stress fracture pain due to the potential of causing imminent fracture, which is very difficult to be controlled by drugs in clinic. In many patients with no clear symptoms, patients usually have no idea that they have been suffering from advanced bone cancer even if there is a sudden fracture induced by mild external force. The bone of patients with bone cancer pain usually has been damaged accompanied by some symptoms before pathological fracture. However, most of these symptoms have been ignored or considered as growing pain. Once pathological fracture occurred, patients with bone cancer may suffer a sudden and severe pain and may suffer a difficulty walking caused by the pain. 


\section{Prevention and treatment of bone cancer pain \\ Prevention of bone cancer pain caused by pathological fractures \\ External radiation ${ }^{31-33}$}

The radiation therapy could relieve the pain and some other symptoms when the cancer cells perform capillary beds to some parts of the bones. The radiation therapy can kill cancer cells or slow the proliferation of cancer cells by high-energy rays to achieve the efficacy of alleviating the pain. The common therapeutic regimen for the patients with bone cancer metastasis is concentrated radiation, also called external beam radiation, to prevent fracture initiation before bone thinning.

\section{Surgery}

The bone can be fixed by surgery to prevent bone fractures to help patients with cancer metastasis to ease the pain. If a fracture has occurred, the internal fixation by surgery can help patients suffering from bone cancer pain relieve pain and return to normal activities. Sometimes, the patients cannot undergo the surgery because of the poor physical condition, the complications, and the side effects of cancer treatment. In this situation without performance of internal fixation, an external fixation with a plywood instead may also be able to have some fixed effect to help patients relieve pain. ${ }^{34}$

\section{Bone cement}

The fractures of bone could be fixed by another regimen using polymethacrylate or glue to fix bone quickly. The technique of injecting polymethacrylate into the spinal cord is called vertebroplasty or kyphoplasty, which can help fix the bone and relieve pain in clinic patients with cancer metastasis.

The therapeutic treatments of radiation therapy and internal fixation, external fixation, and bone cement in patients with bone metastasis pain can prevent fracture around the sites of cancer metastasis or help patients ease pain through fixing the pathological fracture site. Accompanied with the therapy of supplement drugs at the same time, it can enhance the patient's self-confidence to achieve an even better therapeutic effect. ${ }^{35,36}$

\section{Drug treatment Nonsteroidal anti-inflammatory drugs}

The mechanism of nonsteroidal anti-inflammatory is to inhibit the activity of COX, which is a key enzyme to catalyze the arachidonic acid into Prostaglandin (PGE), including COX-1/ COX-2. COX-1 expresses in variety of tissues to regulate cell functions. COX-2 is highly expressed in the tumor cells and peripheral macrophages around the tumor cells because of rapid activation of COX-2 in the role of inflammation, growth factor, and tumor stimuli. ${ }^{37}$ Specific COX-2 inhibitors not only hardly affects the action of COX-1 but also plays the role of anti-inflammatory and anti-tumor effect. In the animal model of bone cancer pain, COX-2-specific inhibitors with high selectivity could alleviate cancer pain with a short-term application and could also reduce the bone damage effect of tumor tissue and osteoclasts. Therefore, most clinicians believe that the nonsteroidal anti-inflammatory drugs are still important drugs for bone cancer pain. ${ }^{26}$

\section{Opioids}

Opioid is one of the most effective drugs for cancer pain currently. Opioid drugs combined with opioid receptors to produce their analgesic effect after treatment in patients, and more than $80 \%$ of patients with cancer need to use opioids to improve or control pain. However, the accompanying side effects of opioid drugs such as tolerance, addiction, excitement, drowsiness, constipation, nausea, vomiting, and respiratory depression limit the further application. Clinicians can optimize the opioid analgesic effect from the following two aspects. First, individualized treatment based on pharmacogenomic studies makes the best analgesic effect and minimal adverse reactions. Second, the application of pharmacodynamic and pharmacokinetic studies achieves the best performance of opioids. Clinicians should pay more attention on the first aspect when the patients are suffering from background pain, while clinicians should pay more attention on the second aspect when the patients are suffering from breakthrough pain and abnormal pain. Obviously, the opioid drugs with the properties of the rapid onset of analgesia, rapid achievement of peak plasma concentration, and the rapid decline are more suitable for the treatment of breakthrough pain and allodynia. ${ }^{38}$

\section{Bisphosphonates}

Bisphosphonate drugs were used to cure the hypercalcemia induced by the advanced malignancies at the beginning. Later, clinicians found that the bisphosphonate drugs could reduce cancer-related bone complications. ${ }^{39}$ These drugs can improve the acidic microenvironment of the local tumor bone tissue, thereby reduce the activation of acid-sensing ion channels and alleviate cancer pain ${ }^{17}$ The bisphosphonate drugs should be considered as the treatment drugs when the analgesic drugs and radiation therapy are not effective in the treatment of bone cancer pain. 


\section{Osteoprotegerin}

Osteoprotegerin (OPG) is a negative regulator of osteoclast, which belongs to a soluble TNF receptor family members, combining with OPG ligand (OPGL) ${ }^{20}{ }^{2}$ OPG plays a role of inhibiting bone destruction through the inhibition of the activation effect of RANKL on osteoclasts. OPG can promote osteoclast apoptosis and prevent bone destruction induced by the tumor cells in mouse femur cancer model, which can relieve cancer pain as well as prevent further bone destruction by tumor cells. In some aspects, the expression levels of neurochemical substances can also be reversed by OPG at the spinal cord level. ${ }^{20}$

\section{Development of drugs ET receptor antagonists}

The ET-1 can be secreted by neuronal cells, non-neuronal cells, and tumor cells. ${ }^{40}$ The ET-1 expression level was very high in a mouse calcaneus cancer pain model, which was related to the formation of bone cancer pain. Hyperalgesia appeared in bone cancer pain was in part due to ET-1 selective sensitization and/or activation of the primary afferent nociceptors containing of ET-1 receptor (also called ET A receptor, ETAR), and injection of ETAR antagonist can alleviate these pain behaviors ${ }^{14}$ The ET system drugs, such as ETAR antagonist atrasentan, have been investigated in clinical treatment on the incidence and development of cancer at the present. The ET receptor antagonists may provide new drug choices for bone cancer pain.

\section{Conclusion}

Bone cancer pain is a kind of chronic pain with unique and complicated mechanisms, in which the occurrence mechanism is always the research hotspot and difficulty to scholars. The successful establishment of several kinds of rodent bone cancer pain models in recent years provides a new platform for investigation of the mechanism and treatment of bone cancer pain, which is very important to explore new therapeutic targets in cancer pain and to improve the life quality of patients suffering from bone cancer pain. The existing rodent models of bone cancer pain are limited to several cancer cell lines with the properties of highly metastasis. However, the patients with primary tumors and metastatic cancer may be suffering from varying degrees of pain. Therefore, it is very urgent and necessary to establish much more animal models related to cancer pain according to the different types of cancer pain in clinical treatment in the further studies. Moreover, the clinical drugs to relief cancer pain are not abundant. Hence, development of new drugs to relief cancer pain using different types of old and novel animal cancer pain models is urgent for improveing the life quality of patients with clinical cancer suffering from pain.

\section{Acknowledgments}

This work was supported by grants from the Zhejiang Provincial Nature Science Foundation of the People's Republic of China (No LY14C050003), the National Natural Science Foundation of the People's Republic of China (No 31470071), the New Century 151 Talent Project of Zhejiang Province, the 521 Talent Foundation of Zhejiang Sci-Tech University, and Zhejiang Provincial Top Key Discipline of Biology. The funders had no role in study design, data collection and analysis, decision to publish, or preparation of the paper.

\section{Disclosure}

The authors report no conflicts of interest in this work.

\section{References}

1. Stewart BW, Wild CP. World Cancer Report 2014 (Print). Lyon Cedex: IARC Nonserial Publication; 2014.

2. Turabi A, Plunkett AR. The application of genomic and molecular data in the treatment of chronic cancer pain. J Surg Oncol. 2012;105:494-501.

3. Reale C, Turkiewicz AM, Reale CA. Antalgic treatment of pain associated with bone metastases. Crit Rev Oncol Hematol. 2001;37:1-11.

4. Mercadante S, Arcuri E. Breakthrough pain in cancer patients: pathophysiology and treatment. Cancer Treat Rev. 1998;24:425-432.

5. Schwei MJ, Honore P, Rogers SD, et al. Neurochemical and cellular reorganization of the spinal cord in a murine model of bone cancer pain. J Neurosci. 1999;19:10886-10897.

6. Guise TA, Kozlow WM,Heras-Herzig A PadaleckiSS, YinJJ, ChirgwinJM. Molecular mechanisms of breast cancer metastases to bone. Clin Breast Cancer. 2005;5:S46-S53.

7. Buga S, Sarria JE. The management of pain in metastatic bone disease. Cancer Control. 2012;19:154-166.

8. Li BT, Wong MH, Pavlakis N. Treatment and prevention of bone metastases from breast cancer: a comprehensive review of evidence for clinical practice. J Clin Med. 2014;3:1-24.

9. Borda AP, Charnay-Sonnek F, Fonteyne V, Papaioannou EG. Guidelines on Pain Management and Palliative Care. Arnhem: European Association of Urology (EAU); 2013

10. Pacharinsak C, Beitz A. Animal models of cancer pain. Comp Med. 2008; 58:220-233.

11. Meuser T, Pietruck C, Radbruch L, Stute P, Lehmann KA, Grond S. Symptoms during cancer pain treatment following who-guidelines: a longitudinal follow-up study of symptom prevalence, severity and etiology. Pain. 2001;93:247-257.

12. Mantyh PW. Cancer pain and its impact on diagnosis, survival, and quality of life. Nat Rev Neurosci. 2006;7:797-809.

13. Honore P, Wchwei J, Rogers SD, et al. Cellular and nuerochemical remodeling of the spinal cord in bone cancer pain. Prog Brain Res. 2000; 129:389-397.

14. Wacnik PW, Eikmeier LJ, Ruggles TR, et al. Functional interactions between tumor and peripheral nerve: morphology, algogen identification, and behavioral characterization of a new murine model of cancer pain. J Neurosci. 2001;21:9355-9366.

15. Wacnik PW, Kehl LJ, Trempe TM, Ramnaraine ML, Beitz AJ, Wilcox GL. Tumor implantation in mouse humerus evokes movement-related hyperalgesia exceeding that evoked by intramuscular carrageenan. Pain. 2003;101:175-186. 
16. Medhurst SJ, Walker K, Bowes M, et al. A rat model of bone cancer pain. Pain. 2002;96:129-140.

17. Walker K, Medhurst SJ, Kidd BL, et al. Disease modifying and antinociceptive effects of the bisphosphonate zoledronic acid in a model of bone cancer pain. Pain. 2002;100:219-229.

18. Portenoy RK, Lesage P. Management of cancer pain. Lancet. 1999; 353:1695-1700.

19. Manthyh PW, Clohisy DR, Koltzenburg M, et al. Molecular mechanisms of cancer pain. Nat Rev Cancer. 2002;2:201-209.

20. Honore P, Luger NM, Sabino MA, et al. Osteoprotegerin blocks bone cancer-induced skeletal destruction, skeletal pain and pain-related neurochemical reorganization of the spinal cord. Nat Med 2000; 6:521-528.

21. Peng X, Guo W, Ren T, et al. Differential expression of RANKL/RANK/ OPG system is associated with bone metastasis in human non-small cell lung cancer. PLoS One. 2013;8:e58361.

22. Sluka KA, Rasmussen LA, Edgar NM, et al. Acid-sensing ion channels 3 deficiency increases inflammation but decreases pain behaviors in murine arthritis. Arthritis Rheumatol. 2013;65:1194-1202.

23. Nelson JB, Carducci MA. The role of endothelin-1 and endothelin receptor antagonists in prostate cancer. BJU Int. 2000;85:45-48.

24. Pomonis JD, Rogers SD, Peters CM, Ghilardi JR, Mantyh PW. Expression and localization of endothelin receptors: implications for the involvement of peripheral gliain nociception. J Neurosci. 2001;21: 999-1006.

25. Opree A, Kress M. Involvement of the proinflammatory cytokines tumor necrosis factor- $(\alpha$, IL-1 $(\beta$, and IL- 6 but not IL- 8 in the development of heat hyperalgesia: effects on heat-evoked calcitonin gene-related peptide release from rat skin. The J Neurosci. 2000;20:6289-6293.

26. Sabino MAC, Ghilardi JR, Jongen JLM, et al. Simultaneous reduction in cancer pain, bone destruction, and tumor growth by selective inhibition of cyclooxygenase-2. Cancer Res. 2002;62:7343-7349.

27. Gordon-Williams RM, Dickenson AH. Central neuronal mechanisms in cancer-induced bone pain. Curr Opin Support Palliat Care. 2007;1: 6-10.

28. Luger NM, Honore P, Sabino MAC, et al. Osteoprotegerin diminishes advanced bone cancer pain. Cancer Res. 2001;61:4038-4047.
29. Meuser T, Pietruck C, Radbruch L, Stute P, Lehmann KA, Grond S Symptoms during cancer pain treatment following who-guidelines: a longitudinal follow-up study of symptom prevalence, severity and etiology. Pain. 2001;93:247-257.

30. Mundy G. Metastasis to bone: causes, consequences and therapeutic opportunities. Nat Rev Cancer. 2002;2:584-593.

31. Belfiore G, Tedeschi E, Ronza FM, et al. Radiofrequency ablation of bone metastases induces long-lasting palliation in patients with untreatable cancer. Singapore Med J. 2008;49:565-570.

32. Basile A, Giuliano G, Scuderi V, et al. Cementoplasty in the management of painfulextraspinal bone metastases: our experience. Radiol Med. 2008;113:1018-1028.

33. Belfiore G, Tedeschi E, Ronza FM, et al. Radiofrequency ablation of bone metastases induces long-lasting palliation in patients with untreatable cancer. Singapore Med J. 2008;49:565-570.

34. Suva LJ, Washam C, Nicholas RW, Griffin RJ. Bone metastasis: mechanisms and therapeutic opportunities. Nat Rev Endocrinol. 2011;7 208-218.

35. Bartels RH, van der Linden YM, van der Graaf W. Spinal extradural metastasis: review of current treatment options. CA Cancer J Clini. 2008;58:245-259.

36. Suva LJ, Griffin RJ, Makhoul I. Mechanisms of bone metastases of breast cancer. Endocr Relat Cancer. 2009;16:703-713.

37. Ma X, Yang Q, Wilson KT, Kundu N, Meltzer SJ, Fulton AM. Promoter methylation regulates cyclooxygenase expression in breast cancer. Breast Cancer Res. 2004,6(4):R316-R321.

38. Yamamoto J, Kawamata T, Niiyama Y, Omote K, Namiki A. Downregulation of mu opioid receptor expression within distinct subpopulations of dorsal root ganglion neurons in a murine model of bone cancer pain. Neuroscience. 2008;151:843-853.

39. Ross JR, Saunders Y, Edmonds PM, Patel S, Broadley KE, Johnston SRD. Systematic review of role of bisphosphonates on skeletal morbidity in metastatic cancer. BMJ. 2003;327:469.

40. Peters CM, Lindsay TH, Pomonis JD, et al. Endothelin and the tumorigenic component of bone cancer pain. Neuroscience. 2004;126:1043-1052.
Drug Design, Development and Therapy

\section{Publish your work in this journal}

Drug Design, Development and Therapy is an international, peerreviewed open-access journal that spans the spectrum of drug design and development through to clinical applications. Clinical outcomes, patient safety, and programs for the development and effective, safe, and sustained use of medicines are a feature of the journal, which

\section{Dovepress}

has also been accepted for indexing on PubMed Central. The manuscript management system is completely online and includes a very quick and fair peer-review system, which is all easy to use. Visit http://www.dovepress.com/testimonials.php to read real quotes from published authors. 